\title{
ELECTROMAGNETIC FIELDS IN LINEAR AND NONLINEAR CHIRAL MEDIA: A TIME-DOMAIN ANALYSIS
}

\author{
IOANNIS G. STRATIS AND ATHANASIOS N. YANNACOPOULOS
}

Received 30 September 2002

We present several recent and novel results on the formulation and the analysis of the equations governing the evolution of electromagnetic fields in chiral media in the time domain. In particular, we present results concerning the well-posedness and the solvability of the problem for linear, time-dependent, and nonlocal media, and results concerning the validity of the local approximation of the nonlocal medium (optical response approximation). The paper concludes with the study of a class of nonlinear chiral media exhibiting Kerr-like nonlinearities, for which the existence of bright and dark solitary waves is shown.

\section{Introduction}

Chiral media are isotropic birefringent substances that respond to either electric or magnetic excitation with both electric and magnetic polarizations. Such media have been known since the end of the nineteenth century (e.g., the study of chirality by Pasteur) and find a wide range of applications from medicine to thin film technology. The understanding of the properties of such media, the differences from ordinary dielectrics, and their possible applications requires detailed mathematical modelling. The mathematical modelling of chiral media is done through the modification of the constitutive relations for normal dielectrics. While for a normal dielectric material the electric displacement $D$ depends solely on the electric field $E$, and the magnetic field $B$ depends solely on the magnetic induction $H$, in a chiral medium, $D$ and $B$ depend on a combination of $E$ and $H$, $[9,11]$. In most cases of interest these constitutive laws are nonlocal relations containing $E$ and $H$. This is a common model for time-dispersive chiral media. Also these constitutive laws may be either linear or nonlinear relations of the fields corresponding to the modelling of linear or nonlinear chiral media, respectively.

Most of the mathematical work on chiral media so far treats the time-harmonic case; see [3] and the references therein. It is the aim of this paper to collect and review some recent results as well as to present some novel ones on the mathematical study of linear and nonlinear chiral media in the time domain. The structure of the paper is as follows. 
We first present some general well-posedness results for models of linear nonlocal chiral media. Then, we introduce a well-known local approximation to nonlocal chiral media, the Drude-Born-Fedorov (DBF) approximation, and study its validity. In the case where the medium under consideration presents a periodic spatial structure, with rapidly varying physical parameters, we study the problem of homogenization, exhibiting that the solution of the problem converges to the solution of a related problem for an effective spatially homogeneous medium whose (constant) parameters are determined.

So far, attention has mainly focused on linear media, with or without time dispersion. However, there is a rapidly growing interest on nonlinear chiral media. The study of such systems is still in its initial stages and very little work has been done in this direction; see, for example, $[7,14]$. In the last section we present some recent results on the evolution of electromagnetic fields in chiral media with cubic nonlinearity, in the weak-dispersion, low-chirality limit, where a set of four coupled partial differential equations of the nonlinear Schrödinger (NLS) type for the evolution of the slowly varying envelopes of the electromagnetic fields is derived and the existence in certain limits of vector solitons of the dark-bright type is established.

\section{Formulation of the problem for linear media}

In this section, we establish the equations governing the evolution of electromagnetic fields in chiral media.

We will start with the Maxwell postulates for a general medium; see, for example, [10]. For a chiral material we have the following constitutive relations that connect the various fields

$$
D=\epsilon E+\epsilon_{1} \star E+\zeta \star H, \quad B=\mu H+\mu_{1} \star H+\xi \star E,
$$

where by $\star$ we denote the convolution operator, that is, $\alpha \star U=\int_{0}^{t} \alpha(x, \tau) U(x, t-\tau) d \tau$.

For a linear medium, the condition that the fields $B$ and $D$ are divergence-free is equivalent to the condition that the fields $E$ and $H$ are divergence-free. Thus the equations for the evolution of the fields in a chiral medium will take the form

$$
\begin{aligned}
\operatorname{curl} E & =-\frac{\partial}{\partial t}\left(\mu H+\mu_{1} \star H+\xi \star E\right)+F, \\
\operatorname{curl} H & =\frac{\partial}{\partial t}\left(\epsilon E+\epsilon_{1} \star E+\zeta \star H\right)+G, \\
\operatorname{div} H & =\operatorname{div} E=0,
\end{aligned}
$$

supplemented with the initial conditions

$$
E(x, 0)=0, \quad H(x, 0)=0 .
$$

This initial value problem will be called hereafter Problem I. The above formulation is valid in unbounded space. The problem may be treated also in domains $\Omega$ with sufficiently smooth boundary $\partial \Omega$ using a boundary condition that corresponds to the physical situation at hand. We will treat here the boundary condition for a perfect conductor. 
In this case the Maxwell equations will have to be complemented with the boundary conditions [10]

$$
n \times E=0, \quad n \cdot H=0 \quad \text { on } \partial \Omega,
$$

where $n$ is the unit outward normal vector to $\partial \Omega$. We now treat the solvability of this problem. First we write it in a more compact form. We define the matrices

$$
A=\left[\begin{array}{cc}
\epsilon I_{3} & 0 \\
0 & \mu I_{3}
\end{array}\right], \quad K=\left[\begin{array}{cc}
\epsilon_{1} I_{3} & \zeta I_{3} \\
\xi I_{3} & \mu_{1} I_{3}
\end{array}\right],
$$

where $I_{3}$ is the $3 \times 3$ unit matrix and 0 is the zero matrix. We further introduce the sixvector notation

$$
\mathscr{E}=(E, H), \quad \mathscr{D}=(D, B), \quad \mathscr{F}=(F, G),
$$

and the differential operator

$$
L=\left[\begin{array}{cc}
0 & \text { curl } \\
- \text { curl } & 0
\end{array}\right],
$$

where again 0 is the zero $3 \times 3$ matrix. The domain of this operator is taken to be

$$
D(L)=\left\{\Phi \mid \Phi=(\phi, \psi) \in X, \operatorname{curl} \phi \in\left(L^{2}(\Omega)\right)^{3}, \operatorname{curl} \psi \in\left(L^{2}(\Omega)\right)^{3}, n \times \phi=0 \text { on } \partial \Omega\right\},
$$

where $X$ is the linear space $X:=L^{2}:=L^{2}(\Omega)^{3} \oplus L^{2}(\Omega)^{3}$ which is a Hilbert space when equipped with the inner product

$$
\langle\mathcal{U}, \mathscr{V}\rangle=\int_{\Omega} \epsilon u_{1} \cdot \bar{v}_{1} d x+\int_{\Omega} \mu u_{2} \cdot \bar{v}_{2} d x=\left\langle\epsilon u_{1}, v_{1}\right\rangle_{0}+\left\langle\mu u_{2}, v_{2}\right\rangle_{0},
$$

where $\mathscr{U}, \mathscr{V} \in L^{2}$, with $\mathscr{U}=\left(u_{1}, u_{2}\right), \mathscr{V}=\left(v_{1}, v_{2}\right)$, and the overbar denotes complex conjugation.

In this notation, Problem I assumes the form

$$
\frac{d}{d t}(A \mathscr{E}+K \star \mathscr{E})=L \mathscr{E}+\mathscr{F}
$$

which is to be solved for given $\mathscr{F}$ and for homogeneous initial conditions $\mathscr{E}(x, 0)=0$.

We will use the Laplace transform $\hat{u}(s)=\int_{0}^{\infty} u(t) e^{-s t} d t$ defined for a real function $u: \mathbb{R} \rightarrow \mathbb{R}$ and for a complex variable $s=\sigma+i \eta$, provided the integral exists [5]. In the following we denote by $\mathscr{L}(\mathbb{R})$ the (linear) space of functions $u \in L_{\text {loc }}^{1}(\mathbb{R})$ such that supp $u \subset$ $[0, \infty)$, and for which the set

$$
I_{u}=\left\{\sigma \in \mathbb{R}: \int_{0}^{\infty}|u(t)| e^{-\sigma t} d t<\infty\right\}
$$

is not empty. We also define the space

$$
\mathscr{L}_{0}=\left\{\widetilde{U} \in L_{\mathrm{loc}}^{1}\left(\mathbb{R}, L^{2}\right):\|\mathcal{U}(\cdot)\| \in \mathscr{L}(\mathbb{R})\right\} .
$$


In the sequel we impose the following three assumptions on the data of the problem:

(A1) $\epsilon$ and $\mu$ are positive and bounded functions of $x$;

(A2) $\mathscr{F}$ has a well-defined Laplace transform (i.e., $\mathscr{F} \in \mathscr{L}_{0}$ );

(A3) the Laplace transform of $K$ exists and converges to the zero matrix as $\sigma \rightarrow \infty$ in any matrix norm.

We take the Laplace transform of (2.10), and using the properties of the Laplace transform and multiplying by $A^{-1}$ from the left, we obtain

$$
(N-s I) \hat{\mathscr{E}}=s \hat{K}_{0} \hat{\mathscr{E}}_{-} \hat{\mathscr{F}}_{0},
$$

where

$$
N=A^{-1} L, \quad \hat{K}_{0}=A^{-1} \hat{K}, \quad \hat{\mathscr{F}}_{0}=A^{-1} \hat{\hat{F}}
$$

This is an equivalent form of the original problem (2.10).

To study the solvability of problem (2.13) we must study the properties of the differential operator $N$. The domain of this operator is $D[N]=D[L]$ and it can be shown that the operator $N$ is unbounded, densely defined, and $i N$ is selfadjoint. Furthermore, if $\operatorname{Re}(s) \neq 0$, the operator $N-s I$ is invertible and the norm of the inverse satisfies the estimate

$$
\left\|(N-s I)^{-1}\right\| \leq \frac{1}{|\sigma|} .
$$

The proofs of these claims are similar to the ones provided for the case of unbounded domains [6].

So, for $s=\sigma \in \mathbb{R}^{+},(2.13)$ is equivalent to the equation

$$
\hat{\mathscr{E}}=s(N-s I)^{-1} \hat{K}_{0} \hat{\mathscr{E}}^{-}(N-s I)^{-1} \hat{\mathscr{F}}_{0} .
$$

But (2.16) is in the form of a fixed point problem, $T \boldsymbol{U}=\boldsymbol{U}$, for the affine operator $T$ : $L^{2} \rightarrow L^{2}$ where

$$
T U=s(N-s I)^{-1} \hat{K}_{0} U-(N-s I)^{-1} \hat{\mathscr{F}}_{0} .
$$

Using this remark as our starting point we are now in a position to state the main result of this section whose proof can be performed along the same lines as in [6]. In particular, the divergence-free property of $E$ and $H$ follows by taking the projection of $D$ and $B$ on the space $H\left(\operatorname{div} 0, \mathbb{R}^{3}\right) \oplus H\left(\operatorname{div} 0, \mathbb{R}^{3}\right)$. Recall that $H\left(\operatorname{div} 0, \mathbb{R}^{3}\right)=\left\{V \in L^{2}\left(\mathbb{R}^{3}\right), \operatorname{div} V=0\right\}$. For the properties of $H\left(\operatorname{div} 0, \mathbb{R}^{3}\right)$ and related spaces, see, for example, [4].

Theorem 2.1. Under the assumptions (A1), (A2), and(A3), Problem I has a unique solution in $D[N]$.

Problem I in a spatially periodic chiral medium (with rapidly varying physical parameters) has been studied from a rigorous homogenization theory point of view in [2] (where in fact the more general case of bianisotropic media is treated). In that work, it has been shown that the solution of the corresponding problem converges to the solution of 
Problem I for an effective spatially homogeneous medium whose (constant) coefficients are determined.

\section{The optical response approximation}

In the previous section, we treated the full nonlocal set of equations, modelling dispersive chiral media, as far as solvability is concerned. Though the mathematical treatment of the full problem is feasible, in a number of important applications (e.g., in wave propagation or scattering problems), the full nonlocal problem may be cumbersome to handle. Thus, local approximations to the full problem have been proposed, that will keep the general features of chiral media, without the mathematical complications introduced by the nonlocality of the model.

In practice, a very common approximation scheme to the full constitutive relations for the medium is used, where essentially the convolution integrals are truncated to a Taylor series in the derivative of the fields. Using this expansion of the convolution integrals and the Maxwell constitutive relations, we may obtain the so-called DBF constitutive relations for chiral media

$$
D=\epsilon(I+\beta \operatorname{curl}) E, \quad B=\mu(I+\beta \operatorname{curl}) H
$$

where $\beta$ is the chirality measure, considered here as a parameter that will be chosen so that a criterion for optimality is satisfied. This approximation is usually called the $o p$ tical response approximation. For such constitutive relations, the equations for the fields become

$$
\begin{aligned}
\operatorname{curl} \tilde{E} & =-\frac{\partial}{\partial t}\{\mu(I+\beta \operatorname{curl}) \tilde{H}\} \\
\operatorname{curl} \tilde{H} & =\frac{\partial}{\partial t}\{\epsilon(I+\beta \operatorname{curl}) \tilde{E}\}, \\
\operatorname{div} \tilde{E} & =0, \quad \operatorname{div} \tilde{H}=0,
\end{aligned}
$$

supplemented with the initial conditions

$$
\tilde{E}(x, 0)=E_{0}(x), \quad \tilde{H}(x, 0)=H_{0}(x),
$$

and the boundary conditions corresponding to the perfect conductor problem. This problem will be called hereafter Problem II. Its solvability is established in the following theorem.

Theorem 3.1. Under the assumptions $A 1$ and A2, Problem II has a unique solution in $D[N]$ for sufficiently small $\beta$.

In this case the Laplace transformed operator equation is of the form $\Theta L \mathscr{E}=P \mathscr{E}+\mathscr{F}$, where $\Theta$ and $P$ are suitably defined matrix operators depending on $s$ and $\beta$. It can be shown that $\Theta$ is invertible; the rest of the proof is similar to that of Theorem 2.1. 
The solution to Problem II is a commonly used approximation to the full solution of Problem I.

A very popular method of treating electromagnetic problems in the frequency domain is through the use of Beltrami fields. This method has been used for the explicit construction of the solution of Problem II in [1].

Another interesting approach to Problem II is through the use of Moses eigenfunctions [13]. These form a complete orthonormal basis for $L^{2}$ consisting of eigenfunctions of the curl operator.

Specifically, Moses [13] introduced three-dimensional complex vectors $K(x, p ; \lambda)$ with $x, p \in \mathbb{R}^{3}$ which satisfy

$$
\operatorname{curl} K(x, p ; \lambda)=\lambda|p| K(x, p ; \lambda), \quad \lambda=0, \pm 1
$$

that is, $K(x, p ; \lambda)$ are eigenvectors of the curl operator and $\lambda|p|$ are the associated eigenvalues. These fields (that will be called Beltrami-Moses fields) satisfy some interesting orthogonality and completeness relations.

We may now define the fields

$$
Q_{ \pm}(x, t)=\{E \pm i \eta H\}(x, t), \quad \eta=\sqrt{\frac{\mu}{\epsilon}}
$$

which implies that

$$
E(x, t)=\frac{1}{2}\left\{Q_{+}+Q_{-}\right\}(x, t), \quad H(x, t)=\frac{1}{2 i \eta}\left\{Q_{+}-Q_{-}\right\}(x, t) .
$$

Using these fields, we may proceed formally to rewrite Problem II in the following form:

$$
\begin{gathered}
\operatorname{curl} Q_{ \pm}= \pm i \sqrt{\mu \epsilon} \frac{\partial}{\partial t}\left\{(I+\beta \operatorname{curl}) Q_{ \pm}(x, t)\right\} \\
\operatorname{div} Q_{ \pm}(x, t)=0 .
\end{gathered}
$$

The associated initial values are

$$
Q_{ \pm}(x, 0)=E_{0}(x) \pm i \eta H_{0}(x) .
$$

Using these Beltrami-Moses fields as kernels for an integral transform, we may define a generalized Fourier transform for vector functions $\psi(x, t)$, the Beltrami-Moses transform, as follows:

$$
\hat{\psi}(p, t ; \lambda)=\int \overline{K(x, p ; \lambda)} \psi(x, t) d x
$$


The inverse transform is given by the formula

$$
\psi(x, t)=\sum_{\lambda} \int K(x, p ; \lambda) \hat{\psi}(p, t ; \lambda) d p .
$$

Expanding the fields $Q_{ \pm}$in terms of the Moses eigenfunctions and using the property that both of these fields have to be divergence-free, we may reduce Problem II to a set of first-order ordinary differential equations for the field amplitudes corresponding to $\lambda=$ \pm 1 . The electromagnetic fields may be obtained by inversion of the integral transform. This approach is related to the spectral approach to Problem II.

\section{The error of the optical response approximation}

Recall that $(E, H)$ and $(\tilde{E}, \tilde{H})$ are, respectively, the solutions of Problems I and II. We introduce a third problem, the solution of which will furnish the error of the optical response approximation. So, let

$$
w_{E}=E-\tilde{E}, \quad w_{H}=H-\tilde{H} .
$$

After some elementary manipulations, we find that the error of the optical response approximation satisfies the equations

$$
\begin{aligned}
\operatorname{curl} w_{E} & =-\frac{\partial}{\partial t}\left\{\mu w_{H}+\mu_{1} \star w_{H}+\xi \star w_{E}+\mu_{1} \star \tilde{H}+\xi \star \tilde{E}-\mu \beta \operatorname{curl} \tilde{H}\right\}, \\
\operatorname{curl} w_{H} & =\frac{\partial}{\partial t}\left\{\epsilon w_{E}+\epsilon_{1} \star w_{E}+\zeta \star w_{H}+\epsilon_{1} \star \tilde{E}+\zeta \star \tilde{H}-\epsilon \beta \operatorname{curl} \tilde{E}\right\}, \\
\operatorname{curl} \tilde{E} & =-\frac{\partial}{\partial t}\{\mu(I+\beta \operatorname{curl}) \tilde{H}\}, \\
\operatorname{curl} \tilde{H} & =\frac{\partial}{\partial t}\{\epsilon(I+\beta \operatorname{curl}) \tilde{E}\}, \\
\operatorname{div} w_{E} & =\operatorname{div} w_{H}=\operatorname{div} \tilde{E}=\operatorname{div} \tilde{H}=0,
\end{aligned}
$$

supplemented with the initial conditions

$$
w_{E}(x, 0)=0, \quad w_{H}(x, 0)=0, \quad \tilde{E}(x, 0)=E_{0}(x), \quad \tilde{H}(x, 0)=H_{0}(x) .
$$

This problem will be hereafter called Problem III. The solution of Problem III will furnish the error of the optical response approximation for a given solution $(\tilde{E}, \tilde{H})$. Observe that the equations for the approximate fields are decoupled from the equations for the error.

A priori estimates are obtained on the solution of Problem III. This is done by reducing the error equations to the form of a Volterra equation of the second kind. By expanding the solution in Moses eigenfunctions, we may rewrite the original system for the error in the compact form

$$
A_{1} w=\frac{d}{d t}\left\{A_{2} w+A_{3} \star w+S\right\}
$$


where

$$
\begin{aligned}
w & =\left(\begin{array}{c}
w_{E, \lambda} \\
w_{H, \lambda}
\end{array}\right), \quad A_{1}=\left(\begin{array}{cc}
\lambda|p| & 0 \\
0 & \lambda|p|
\end{array}\right), \\
A_{2} & =\left(\begin{array}{cc}
0 & -\mu \\
\epsilon & 0
\end{array}\right), \quad A_{3}=\left(\begin{array}{cc}
-\xi & -\mu_{1}(\tau) \\
\epsilon_{1}(\tau) & \zeta
\end{array}\right), \\
S & =\left(\begin{array}{l}
S_{1, \lambda} \\
S_{2, \lambda}
\end{array}\right)=\left(\begin{array}{c}
-\mu_{1} \star \bar{H}_{\lambda}-\xi \star \bar{E}_{\lambda}+\lambda \beta \mu|p| \bar{H}_{\lambda} \\
\epsilon_{1} \star \bar{E}_{\lambda}+\zeta \star \bar{H}_{\lambda}-\lambda \beta \epsilon|p| \bar{E}_{\lambda}
\end{array}\right) .
\end{aligned}
$$

Now integrate once over time to rewrite the equation for the error in the following form:

$$
w=\phi \star w+g
$$

where

$$
\phi=A_{2}^{-1}\left(A_{1}-A_{3}\right), \quad g=-A_{2}^{-1} S
$$

For the specific system we study here, we have that

$$
\phi=\left(\begin{array}{cc}
-\frac{\epsilon_{1}(\tau)}{\epsilon} & \frac{\lambda|p|-\zeta}{\epsilon} \\
-\frac{\lambda|p|+\xi}{\mu} & -\frac{\mu_{1}(\tau)}{\mu}
\end{array}\right), \quad g=\left(\begin{array}{c}
\frac{\epsilon_{1}}{\epsilon} \star \bar{E}_{\lambda}+\frac{\zeta}{\epsilon} \star \bar{H}_{\lambda}-\lambda \beta|p| \bar{E}_{\lambda} \\
\frac{\mu_{1}}{\mu} \star \bar{H}_{\lambda}+\frac{\xi}{\mu} \star \bar{E}_{\lambda}-\lambda \beta|p| \bar{H}_{\lambda}
\end{array}\right)
$$

This matrix Volterra equation will be used to obtain a priori estimates for the error of the optical response approximation in terms of the Moses transformed fields. The following two results were proved in [6].

Theorem 4.1. Let

$$
\Psi(t):=\left(1-2 \sup _{i, j}\left\|\phi_{i j}\right\|_{L_{1}(0, t)}\right)^{-1}>0 .
$$

Then, the solution of (4.6) satisfies the following a priori error bound

$$
\sup _{i}\left\|w_{i}\right\|_{L_{p}(0, t)} \leq \Psi(t) \sup _{i}\left\|g_{i}\right\|_{L_{p}(0, t)}
$$

It is interesting to notice that an alternative method of obtaining a priori bounds can be developed using the Gronwall inequality. Indeed, in this manner we can readily obtain the following result.

Theorem 4.2. Suppose that $\epsilon>0, \mu>0, \xi>0$, and $\zeta>0$, and that $|p| \leq \min (\xi, \zeta)$. Assuming that the functions $\phi_{i j}$ are bounded, the following estimate holds:

$$
\sup _{t}\left|w_{1}(t)+w_{2}(t)\right| \leq \sup _{t}\left|g_{1}(t)+g_{2}(t)\right| \text {. }
$$


The above estimate provides us with a way to minimize the error of the optical response approximation. One way to do this is by minimizing the upper bound $\sup _{i}\left\|g_{i}\right\|_{L_{r}(0, t)}$. This amounts to choosing the value of $\beta$ so as to minimize the integrals

$$
\begin{aligned}
& \left\|g_{1}\right\|_{L_{r}(0, t)}=\left\{\int_{0}^{t}\left|\frac{\epsilon_{1}}{\epsilon} \star \bar{E}_{\lambda}+\frac{\zeta}{\epsilon} \star \bar{H}_{\lambda}-\lambda \beta\right| p\left|\bar{E}_{\lambda}\right|^{r} d t^{\prime}\right\}^{1 / r}, \\
& \left\|g_{2}\right\|_{L_{r}(0, t)}=\left\{\int_{0}^{t}\left|\frac{\mu_{1}}{\mu} \star \bar{H}_{\lambda}+\frac{\xi}{\mu} \star \bar{E}_{\lambda}-\lambda \beta\right| p\left|\bar{H}_{\lambda}\right|^{r} d t^{\prime}\right\}^{1 / r} .
\end{aligned}
$$

A series of other results were obtained for each $p$ using the expansion of Problem III in Moses eigenfunctions. This approach allows us to find exact forms for the Laplace transform of the error for specified wavenumbers. Numerical techniques can thus be used for the inversion of the Laplace transform and the retrieval of the time dependence of the error term.

An estimate of the error in the spatial variables rather than in terms of the wavenumbers can be obtained in the following way. Adopting the notation of Section 2, the equation for the error may be written in the form

$$
L w=\frac{\partial}{\partial t}(A w+K \star w+\Phi)
$$

where $\Phi$ is a source term which is related to the solutions of the optical response equation $\tilde{H}$ and $\tilde{E}$. Multiplying by $w$, integrating over space, and using the properties of the operator $L$, we obtain

$$
\frac{1}{2} \frac{d}{d t}\left(\|w\|^{2}\right)+\left\langle\frac{d}{d t}(K \star w), w\right\rangle+\langle\Phi, w\rangle
$$

Under the assumption that the convolution kernel is such that

$$
K_{1} A w \leq \frac{d}{d t}(K \star w) \leq K_{2} A w
$$

we obtain

$$
\frac{d}{d t}\|w\|^{2}+K_{1}\|w\|^{2} \leq|\Phi|\|w\|
$$

from which by use of the Gronwall inequality we may obtain a priori bounds for the error. Similar bounds may be obtained by slight modification of the conditions on the kernels.

\section{Homogenization for spatially periodic chiral media}

We will now consider Problem I in a spatially periodic chiral medium, that is, we will consider the parameters of the medium $\epsilon, \epsilon_{1}, \mu, \mu_{1}, \zeta$, and $\xi$ to be periodic functions of $x$ with a period $\varepsilon$. The period $\varepsilon$ will be considered to be a small number, a fact that will correspond to a fast spatially varying medium. 
This leads us to considering the problem of homogenization for such media, that is, the approximation of a spatially periodic medium with a homogeneous medium (i.e., a medium with constant parameters) having the same properties as the original medium in the limit as $\varepsilon \rightarrow 0$. The homogenization problem for periodic structures is a long standing problem in the mathematical and engineering community that has led to the introduction of interesting mathematical techniques and also to interesting engineering applications.

Though the problem of homogenization for the Maxwell equations has been studied extensively in the past, there has been little progress on this subject as far as bianisotropic or chiral media are concerned. While some papers treat versions of the problem from the engineering point of view, the problem has been left untouched from the rigorous mathematical point of view. This aspect of the problem has been studied in [2] for the more general case of bianisotropic media.

Consider the spatially periodic version of Problem I, which consists of Maxwell's equations

$$
\begin{gathered}
\partial_{t} D^{\varepsilon}=\operatorname{curl} H^{\varepsilon}+F(x, t), \\
\partial_{t} B^{\varepsilon}=-\operatorname{curl} E^{\varepsilon}+G(x, t), \quad x \in \Omega, t>0, \\
E^{\varepsilon}(x, 0)=0, \quad H^{\varepsilon}(x, 0)=0, \quad x \in \Omega,
\end{gathered}
$$

subject to the constitutive laws

$$
\begin{aligned}
D^{\varepsilon} & =\epsilon^{\varepsilon} E^{\varepsilon}+\zeta^{\varepsilon} * H^{\varepsilon}+\epsilon_{1}^{\varepsilon} * E^{\varepsilon}, \\
B^{\varepsilon} & =\mu^{\varepsilon} H^{\varepsilon}+\xi^{\varepsilon} * E^{\varepsilon}+\mu_{1}^{\varepsilon} * H^{\varepsilon} .
\end{aligned}
$$

The functions $\epsilon^{\varepsilon}(x)$ and $\mu^{\varepsilon}(x)$ as well as the functions $\epsilon_{1}^{\varepsilon}(x, t), \mu_{1}^{\varepsilon}(x, t), \xi^{\varepsilon}(x, t)$, and $\zeta^{\varepsilon}(x, t)$ are periodic in $x$ of period $\varepsilon Y$. We assume that there exists $c>0$ such that the block matrix

$$
\left(\begin{array}{cc}
\epsilon+\hat{\epsilon}_{1} & \hat{\zeta} \\
\hat{\xi} & \mu+\hat{\mu}_{1}
\end{array}\right)=: A(x, p)
$$

satisfies

$$
\langle A(x, p) U, U\rangle \geq c\|U\|^{2}, \quad x \in \Omega, p \in \mathbb{C}_{+}, U \in \mathbb{R}^{6} .
$$

We fix a domain $V \subset \Omega$ and consider the operator

$$
L^{\varepsilon}=\left(\begin{array}{cc}
-\operatorname{div}\left(\left(\epsilon+\hat{\epsilon}_{1}\right) \operatorname{grad}\right) & -\operatorname{div}((\hat{\zeta}) \operatorname{grad}) \\
-\operatorname{div}((\hat{\xi}) \operatorname{grad}) & -\operatorname{div}\left(\left(\mu+\hat{\mu}_{1}\right) \operatorname{grad}\right)
\end{array}\right): H_{0}^{1}(V) \longrightarrow H^{-1}(V)
$$

and the corresponding homogenization limit

$$
L^{h}=:\left(\begin{array}{ll}
-\operatorname{div}\left(\tilde{\epsilon}^{h} \operatorname{grad}\right) & -\operatorname{div}\left(\tilde{\zeta}^{h} \operatorname{grad}\right) \\
-\operatorname{div}\left(\tilde{\xi}^{h} \mathrm{grad}\right) & -\operatorname{div}\left(\tilde{\mu}^{h} \mathrm{grad}\right)
\end{array}\right) .
$$


Note that while the coefficients of $L^{h}$ are spatially constant, they do depend on $p \in \mathbb{C}_{+}$. We assume that for fixed $x \in \Omega$, the functions $\tilde{\epsilon}^{h}, \tilde{\xi}^{h}, \tilde{\zeta}^{h}$, and $\tilde{\mu}^{h}$ are the Laplace transforms of functions $\epsilon^{h}, \xi^{h}, \zeta^{h}$, and $\mu^{h}$ on $(0, \infty)$. We then have the following theorem.

Theorem 5.1. Assume that the Maxwell system (5.1) and (5.2) is uniquely solvable for all $\varepsilon>0$ and that $\left\|E^{\varepsilon}\right\|_{2},\left\|H^{\varepsilon}\right\|_{2} \leq c$ for all $\varepsilon, t>0$. Then the solution $\left(E^{\varepsilon}, H^{\varepsilon}\right)$ of the above system satisfies

$$
E^{\varepsilon} \longrightarrow E^{*}, \quad H^{\varepsilon} \longrightarrow H^{*}, \quad * \text {-weakly in } L^{\infty}\left((0, \infty), L^{2}(\Omega)\right)
$$

where $\left(E^{*}, H^{*}\right)$ is the unique solution of the Maxwell system

$$
\begin{gathered}
\partial_{t} D^{*}=\operatorname{curl} H^{*}+F, \\
\partial_{t} B^{*}=-\operatorname{curl} E^{*}+G, \quad x \in \Omega, \quad t>0, \\
E^{*}(x, 0)=0, \quad H^{*}(x, 0)=0,
\end{gathered}
$$

subject to the constitutive laws

$$
\begin{aligned}
& D^{*}=\epsilon^{h} * E^{*}+\zeta^{h} * H^{*}, \\
& B^{*}=\xi^{h} * E^{*}+\mu^{h} * H^{*} .
\end{aligned}
$$

We do not provide the proof of the theorem here (for a complete proof see [2]) but simply note that in order to prove the above result, we have to work with the Laplace transform of the original problem which assumes the form of an elliptic partial differential equation with spatially periodic coefficients. The homogenization problem for the latter may be addressed using generalizations of standard homogenization techniques based on the use of the div-curl lemma, thus leading to a spatially homogenized equation in Laplace space. Then, inverting the Laplace transform, we arrive at the announced result. For details see [2].

Remark 5.2. (1) The above theorem gives the homogenized coefficients as inverse Laplace transforms of certain functions. In concrete cases one can use numerical schemes to obtain precise approximations of $\epsilon^{h}, \xi^{h}, \zeta^{h}$, and $\mu^{h}$. The Laplace transforms of the homogenized coefficients may be obtained by a proper averaging of the parameters of the medium weighted by the solution of an appropriately formulated "cell problem." For the definition of the cell problem see [2].

(2) It is clear that the functions $F$ and $G$ can also depend on $\varepsilon>0$, provided that one makes suitable assumptions on their behaviour as $\varepsilon \rightarrow 0$.

For completeness here, we present the expressions for the homogenized coefficients for the medium, in Laplace space.

We let $H_{\mathrm{per}}^{1}(Y)$ denote the closed subspace of $H^{1}(Y)$ that consists of periodic functions and define the operator $L_{\text {per }}: H_{\text {per }}^{1}(Y) \rightarrow\left(H_{\text {per }}^{1}(Y)\right)^{*}$ by

$$
L_{\mathrm{per}}=\left(\begin{array}{ll}
-\operatorname{div}\left(\epsilon I_{3} \operatorname{grad}\right) & -\operatorname{div}\left(\zeta I_{3} \operatorname{grad}\right) \\
-\operatorname{div}\left(\xi I_{3} \operatorname{grad}\right) & -\operatorname{div}\left(\mu I_{3} \operatorname{grad}\right)
\end{array}\right) .
$$


This operator may be proved to be invertible modulo constants. In particular, we can define (modulo constants) the functions $u_{1}^{j}, u_{2}^{j}, v_{1}^{j}$, and $v_{2}^{j}, j=1,2,3$, by the relations

$$
L_{\text {per }}\left(\begin{array}{c}
u_{1}^{j} \\
u_{2}^{j}
\end{array}\right)=\left(\begin{array}{c}
\frac{\partial \epsilon_{i j}}{\partial y_{i}} \\
\frac{\partial \xi_{i j}}{\partial y_{i}}
\end{array}\right), \quad L_{\text {per }}\left(\begin{array}{c}
v_{1}^{j} \\
v_{2}^{j}
\end{array}\right)=\left(\begin{array}{c}
\frac{\partial \zeta_{i j}}{\partial y_{i}} \\
\frac{\partial \mu_{i j}}{\partial y_{i}}
\end{array}\right) .
$$

We define the homogenized constant coefficient matrices $\epsilon^{h}, \xi^{h}, \zeta^{h}$, and $\mu^{h}$ by

$$
\begin{aligned}
\epsilon_{i j}^{h} & =\left\langle\epsilon_{i j}+\epsilon_{i k} \partial_{y_{k}} u_{1}^{j}+\zeta_{i k} \partial_{y_{k}} u_{2}^{j}\right\rangle, \\
\xi_{i j}^{h} & =\left\langle\xi_{i j}+\xi_{i k} \partial_{y_{k}} u_{1}^{j}+\mu_{i k} \partial_{y_{k}} u_{2}^{j}\right\rangle, \\
\zeta_{i j}^{h} & =\left\langle\zeta_{i j}+\zeta_{i k} \partial_{y_{k}} v_{2}^{j}+\epsilon_{i k} \partial_{y_{k}} v_{1}^{j}\right\rangle, \\
\mu_{i j}^{h} & =\left\langle\mu_{i j}+\mu_{i k} \partial_{y_{k}} v_{2}^{j}+\xi_{i k} \partial_{y_{k}} v_{1}^{j}\right\rangle,
\end{aligned}
$$

where $\langle g\rangle:=|Y|^{-1} \int_{Y} g$. It is not obvious but it is easy to prove that the block matrix

$$
A^{h}=\left(\begin{array}{ll}
\epsilon^{h} & \zeta^{h} \\
\xi^{h} & \mu^{h}
\end{array}\right)
$$

is symmetric and positive definite. We note here that one can also deduce relations (5.12) formally by postulating a double-scale expansion for $E^{\varepsilon}$ and $H^{\varepsilon}$.

\section{Nonlinear chiral media}

The results presented so far were results valid for chiral media constitutive relations with linear (local or nonlocal) laws involving the electromagnetic fields. Nevertheless there is a rapidly growing interest in nonlinear chiral media. The study of such systems is still in its initial stages and very little work has been done in this direction (see, e.g., [7, 14]). In this section we will examine the effects of nonlinearity on the constitutive relations for chiral media. In particular, we will present some recent results related to the evolution of electromagnetic fields in chiral media with cubic nonlinearity in the weak-dispersion, low-chirality limit. This limit is quite interesting and has been studied in the linear case (for general mathematical results for time-harmonic fields see, e.g., [3] and the references therein). For cubically nonlinear, weakly dispersive media with low-chirality parameter, we derive a set of four coupled partial differential equations of the NLS type for the evolution of the slowly varying envelopes of the electromagnetic fields. With the use of reductive perturbation theory, we reduce the system to a set of integrable partial differential equations, the Mel'nikov system, and thus show the existence in certain limits of vector solitons of the dark-bright type.

6.1. The field equations in the general case. The starting point for the modelling of a nonlinear chiral medium is the Maxwell postulates, in the absence of sources. We assume furthermore that the medium is of infinite extent. To obtain a description of the fields, 
the above equations will have to be complemented by the constitutive relations for the medium, that give the connection of $D$ and $B$ on the fields $E$ and $H$. For a weakly nonlinear, weakly dispersive chiral medium with a cubic nonlinearity, we may assume that the constitutive relations in the time domain are of the form

$$
\begin{aligned}
& D=\epsilon E+\epsilon_{1} \star E+\zeta \star H+\delta \epsilon_{2} f_{1}\left(|E|^{2}\right) E \\
& B=\mu H+\mu_{1} \star H+\xi \star E+\delta \mu_{2} f_{2}\left(|H|^{2}\right) H
\end{aligned}
$$

where by $\star$ we denote the convolution $(f \star G)(x, t)=\int_{-\infty}^{\infty} f\left(t-t^{\prime}\right) G\left(x, t^{\prime}\right) h\left(t-t^{\prime}\right) d t^{\prime}$. In the above relation, $\epsilon$ and $\mu$ are the permittivity and permeability of the medium, respectively, and $\xi$ and $\zeta$ are the chirality parameters of the medium. Causality in the linear part is ensured by the appearance of the Heaviside function $h$ whereas the nonlinear part is local (and therefore causal). The assumption of locality for the nonlinear part is consistent with the weak dispersion-the weak nonlinearity case we consider. The parameter $\delta$ is a small parameter which is associated with the weak nonlinearity. The fact that we have low-chirality and weak nonlinearity is shown in the above constitutive relations by the fact that the nonlinearity in $D$ depends only on $E$ while the nonlinearity in $B$ depends only on $H$. We further assume that the chirality effects are weaker than the nonlinearity so as to be able to neglect cross terms in the fields $H$ and $E$. In this work we use constitutive relations with nonlinearities expressed directly in the fields $E$ and $H$, and not in the Beltrami fields in which they may be decomposed (see, e.g., [14]).

The well-posedness of the above problem in the general case is an intriguing mathematical problem which is currently under consideration. Here we will study the problem for a special class of fields, that is, fields which in the frequency domain are of the form

$$
\begin{gathered}
E(z, \omega)=u_{1}(z, \omega) e_{+}+v_{1}(z, \omega) e_{-}, \\
H(z, \omega)=u_{2}(z, \omega) e_{+}+v_{2}(z, \omega) e_{-},
\end{gathered}
$$

where $e_{ \pm}=(1 / \sqrt{2})(\hat{x} \pm i \hat{y})$. This ansatz contains the most general dependence of the fields in $e_{+}$and $e_{-}$(which is a complete basis in the $x, y$ plane). On the other hand it does not contain the longitudinal component and/or transverse dependence of the fields, nevertheless it is still consistent with the divergence-free property of the fields $D$ and $B$ which is valid in this case.

Substituting this ansatz in the Maxwell postulates, we arrive at the following set of nonlinear equations (in the frequency domain):

$$
\begin{aligned}
-i \frac{\partial u_{1}}{\partial z} & =-i \omega\left(\mu u_{2}+\hat{\mu}_{1} u_{2}+\hat{\zeta} u_{1}+\delta \mu_{2} f_{2}\left(\left|u_{2}\right|^{2}+\left|v_{2}\right|^{2}\right) u_{2}\right) \\
-i \frac{\partial u_{2}}{\partial z} & =i \omega\left(\epsilon u_{1}+\hat{\epsilon}_{1} u_{1}+\hat{\xi} u_{2}+\delta \epsilon_{2} f_{1}\left(\left|u_{1}\right|^{2}+\left|v_{1}\right|^{2}\right) u_{1}\right) \\
i \frac{\partial v_{1}}{\partial z} & =-i \omega\left(\mu v_{2}+\hat{\mu}_{1} v_{2}+\hat{\zeta} v_{1}+\delta \mu_{2} f_{2}\left(\left|u_{2}\right|^{2}+\left|v_{2}\right|^{2}\right) v_{2}\right) \\
i \frac{\partial v_{2}}{\partial z} & =i \omega\left(\epsilon v_{1}+\hat{\epsilon}_{1} v_{1}+\hat{\xi} v_{2}+\delta \epsilon_{2} f_{1}\left(\left|u_{1}\right|^{2}+\left|v_{1}\right|^{2}\right) v_{1}\right)
\end{aligned}
$$


where by $\hat{\phi}(\omega)$ we denote the Fourier transform of $\phi(t)$ (the $\omega$ dependence is dropped for convenience). We note that in the above set of equations in the absence of nonlinearity $(\delta=0)$, the first two equations decouple from the other two, that is, the field components in the $\mathbf{e}_{+}$and $\mathbf{e}_{-}$directions evolve independently. The components, in the absence of boundary conditions, are coupled only through the nonlinearity.

6.2. Derivation of the amplitude equations. Assuming solutions of the form

$$
u_{j}(z, \omega)=U_{j} \exp \left(i k_{+} z\right), \quad v_{j}(z, \omega)=V_{j} \exp \left(i k_{-} z\right), \quad j=1,2,
$$

which in the time domain correspond to wave solutions, we see that in the weakly nonlinear case they will have to satisfy the dispersion relations

$$
\begin{gathered}
k_{ \pm}^{2} \pm i \omega(\hat{\zeta}-\hat{\xi}) k_{ \pm}+\omega^{2}\left\{\hat{\xi} \hat{\zeta}-\left(\epsilon+\hat{\epsilon}_{1}+\delta \epsilon_{2} f_{1}\right)\left(\mu+\hat{\mu}_{1}+\delta \mu_{2} f_{2}\right)\right\}=0 \\
f_{i}=f_{i}\left(\left|U_{i}\right|^{2}+\left|V_{i}\right|^{2}\right), \quad i=1,2 .
\end{gathered}
$$

In the absence of nonlinearity $(\delta=0)$, these two dispersion relations reduce to the dispersion relations for the right-handed and the left-handed polarized waves that are well known to propagate in linear chiral media.

We will now assume that the nonlinear medium supports wave solutions of the form (6.4), where the Fourier transforms of $U_{j}$ and $V_{j}$ are considered to be slowly varying functions of space and time. In other words, $U_{j}$ and $V_{j}$ are considered to be the envelopes of the wave fields. Using reductive perturbation theory, we may derive modulation equations for the evolution of the envelopes of the fields. One way of doing that is through the dispersion relation of the weakly nonlinear waves in the following way: we expand the dispersion relations in a Taylor expansion around the point $\left(k_{0}, \omega_{0}\right)$ which is a solution of the linear dispersion relation. For the problem at hand, it is enough to keep terms up to the second order. As a result we obtain a polynomial expression in $k$ and $\omega$, and $I_{i}=\left|U_{i}\right|^{2}+\left|V_{i}\right|^{2}, i=1,2$, the coefficients of the polynomial, are derivatives of $\omega$ calculated at the points $k=k_{0}$ and $I_{i}=0$ (linear case).

In order to obtain modulation equations for the envelopes of the fields, we have to return back to physical space and time. This is done through the substitution $\left(\omega_{ \pm}-\omega_{0, \pm}\right) \rightarrow$ $i(\partial / \partial t),\left(k-k_{0}\right) \rightarrow-i(\partial / \partial z)$, and assuming that these operators act on the envelope of the waves and on the relevant temporal and spatial scales. The modulation equations may be derived in an alternative manner by the use of reductive perturbation theory.

Following the procedure described above, we obtain a set of four evolution equations for the envelopes of the wave fields, in the time domain, of the form

$$
\begin{aligned}
& i \frac{\partial U_{j}}{\partial t}=i \frac{\partial \omega_{+}}{\partial k} \frac{\partial U_{j}}{\partial z}+\frac{1}{2} \frac{\partial^{2} \omega_{+}}{\partial k^{2}} \frac{\partial^{2} U_{j}}{\partial z^{2}}+\left(\frac{\partial \omega_{+}}{\partial I_{1}} I_{1}+\frac{\partial \omega_{+}}{\partial I_{2}} I_{2}\right) U_{j} \\
& i \frac{\partial V_{j}}{\partial t}=i \frac{\partial \omega_{-}}{\partial k} \frac{\partial V_{j}}{\partial z}+\frac{1}{2} \frac{\partial^{2} \omega_{-}}{\partial k^{2}} \frac{\partial^{2} V_{j}}{\partial z^{2}}+\left(\frac{\partial \omega_{-}}{\partial I_{1}} I_{1}+\frac{\partial \omega_{-}}{\partial I_{2}} I_{2}\right) V_{j}
\end{aligned}
$$

where $j=1,2$. These NLS equations are coupled through the nonlinear terms $I_{1}$ and $I_{2}$. The spatial and temporal coordinates appearing in these equations are scaled variables, 
relevant to the slow variations of the envelopes of the fields. For more details on the derivation of the modulation equations see [7].

6.3. Reduction of the modulation equations to the Mel'nikov system. The coupled NLS equations which arose as modulation equations for the evolution of the fields in chiral media are not integrable by the use of the inverse scattering transform. However, it is possible, through the use of reductive perturbation theory, to reduce the system in the proper spatial and time scales to an integrable system that approximates the behaviour of the original system. This procedure is a general way of understanding the properties of solutions of nonintegrable systems that has been proven fruitful in a number of similar situations (see [7] and the references therein).

To obtain the reduction to an integrable system, we restrict ourselves to solutions of the type

$$
U_{2}=\rho_{1} U_{1}, \quad V_{2}=\rho_{2} V_{1}
$$

for which the original system of NLS equations reduces to a system of two equations.

We will now look for solutions of the above system satisfying the boundary conditions

$$
\begin{aligned}
& \left|U_{1}\right| \longrightarrow|u|, \quad \text { as } z \longrightarrow \infty, \\
& \left|V_{1}\right| \longrightarrow 0, \quad \text { as } z \longrightarrow \infty
\end{aligned}
$$

that is, we will look for solutions of the dark soliton type in the right-handed component $u$, and solutions of the bright soliton type in the left-handed component $v$. It is clear that the above boundary conditions may be reversed.

With the use of reductive perturbation theory (for details see [7]), a lengthy procedure leads to a system of the Mel'nikov type [12], which is fully integrable by the inverse scattering transform for special cases of the parameters. The Mel'nikov system has soliton solutions in the form of a dark soliton in the right-handed component and a bright soliton in the left-handed component, that is, a localized nonlinear wave propagating in a dispersive medium, on top of a continuous wave background, keeping its shape undistorted. The bright soliton represents a bulge on top of the continuous wave background, whereas the dark soliton represents a dip. For more details on the definition and the properties of dark and bright solitons see, for example, [8].

\section{Acknowledgment}

The authors acknowledge the partial financial support from the Special Research Account of the University of Athens (Grant 70/4/5643) and the second author acknowledges the partial support from the National Scholarship Foundation Postdoctoral Programme.

\section{References}

[1] C. Athanasiadis, G. F. Roach, and I. G. Stratis, A time domain analysis of wave motions in chiral materials, Math. Nachr. 250 (2003), 3-16.

[2] G. Barbatis and I. G. Stratis, Homogenization of Maxwell's equations in dissipative bianisotropic media, Math. Methods Appl. Sci. 26 (2003), no. 14, 1241-1253. 
[3] S. Bernstein, K. Gürlebeck, K. Khmelnytskaya, V. Kravchenko, H. Oviedo, and I. G. Stratis, Electromagnetic Fields in Chiral Media: Vector and Quaternionic Approach, under review.

[4] R. Dautray and J.-L. Lions, Mathematical Analysis and Numerical Methods for Science and Technology. Vol. 3, Spectral Theory and Applications, Springer-Verlag, Berlin, 1990.

[5] _ Mathematical Analysis and Numerical Methods for Science and Technology Vol. 5, Evolution Problems I, Springer-Verlag, Berlin, 1992.

[6] D. J. Frantzeskakis, A. Ioannidis, G. F. Roach, I. G. Stratis, and A. N. Yannacopoulos, On the error of the optical response approximation in chiral media, Appl. Anal. 82 (2003), no. 9, 839-856.

[7] D. J. Frantzeskakis, I. G. Stratis, and A. N. Yannacopoulos, Bright-dark vector solitons in chiral media, Phys. Scripta 66 (2002), 280-283.

[8] Yu. S. Kivshar and B. Luther-Davies, Dark optical solitons: physics and applications, Phys. Rep. 298 (1998), 81-197.

[9] A. Lakhtakia, Beltrami Fields in Chiral Media, World Scientific, London, 1994.

[10] R. Leis, Initial-Boundary Value Problems in Mathematical Physics, B. G. Teubner, Stuttgart; John Wiley \& Sons, Chichester, 1986.

[11] I. V. Lindell, A. H. Sihvola, S. A. Tretyakov, and A. J. Viitanen, Electromagnetic Waves in Chiral and Bi-isotropic Media, Artech House, Massachusetts, 1994.

[12] V. K. Mel'nikov, Exact solutions of the Korteweg-de Vries equation with a self-consistent source, Phys. Lett. A 128 (1988), no. 9, 488-492.

[13] H. E. Moses, Eigenfunctions of the curl operator, rotationally invariant Helmholtz theorem, and applications to electromagnetic theory and fluid mechanics, SIAM J. Appl. Math. 21 (1971), 114-144.

[14] G. Ya. Slepyan, S. A. Maksimenko, F. G. Bass, and A. Lakhtakia, Nonlinear electromagnetics in chiral media: self-action of waves, Phys. Rev. E (3) 52 (1995), 1049-1058.

Ioannis G. Stratis: Department of Mathematics, University of Athens, Panepistimiopolis, Zographou, 15784 Athens, Greece

E-mail address: istratis@math.uoa.gr

Athanasios N. Yannacopoulos: Department of Statistics and Actuarial Science, University of the Aegean, Karlovassi, 83200 Samos, Greece

E-mail address: ayannaco@aegean.gr 


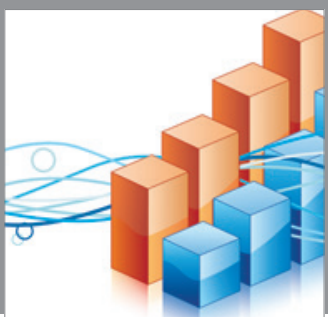

Advances in

Operations Research

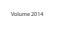

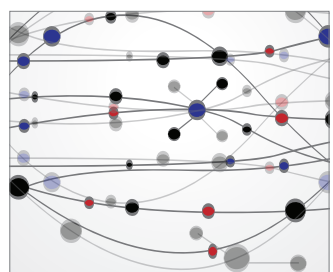

\section{The Scientific} World Journal
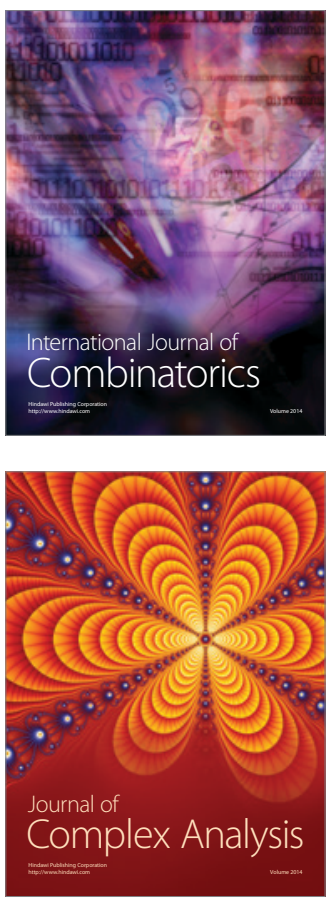

International Journal of

Mathematics and

Mathematical

Sciences
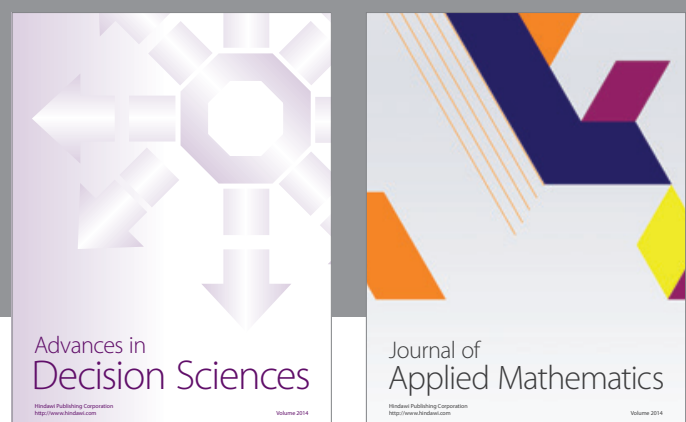

Journal of

Applied Mathematics
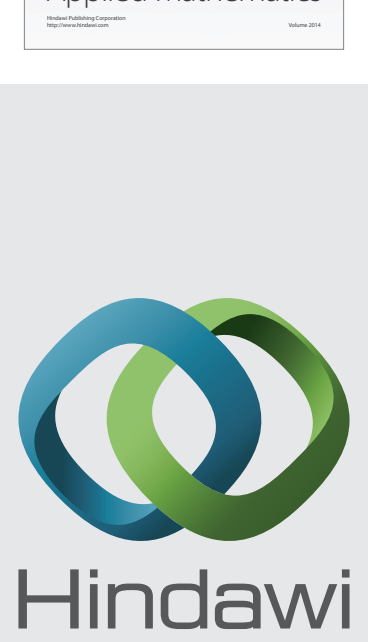

Submit your manuscripts at http://www.hindawi.com
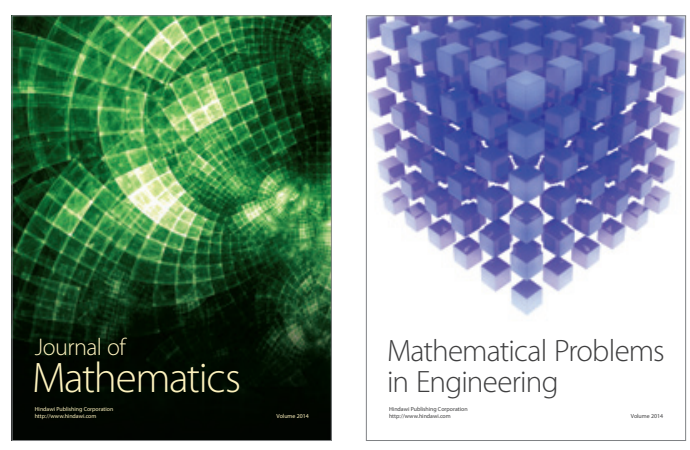

Mathematical Problems in Engineering
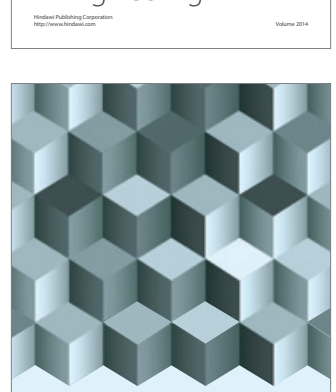

Journal of

Function Spaces
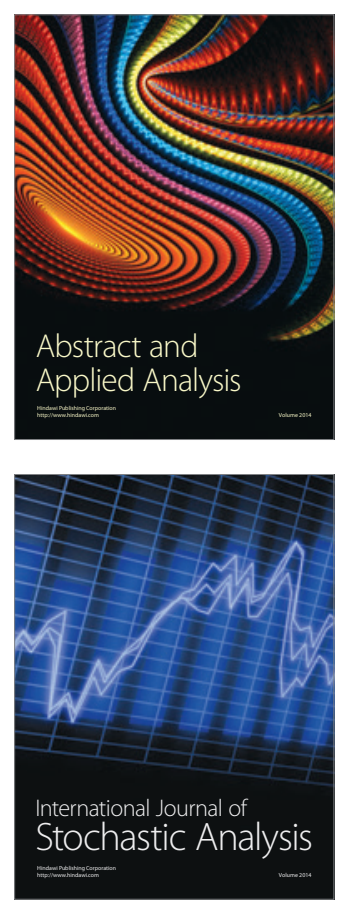

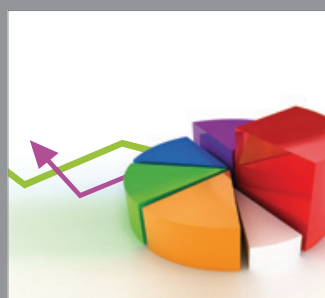

ournal of

Probability and Statistics

Promensencen
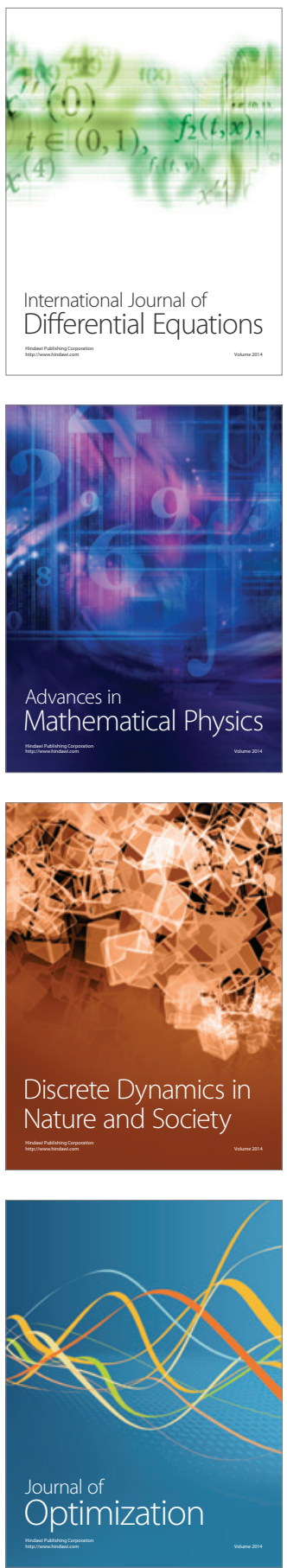